\title{
Avaliação dos impactos das precipitações pluviométricas na manutenção da rede de esgotamento sanitário no Município de Caruaru, Agreste de Pernambuco
}

\section{José Floro de Arruda Neto ${ }^{1}, *$, Iálysson da Silva Medeiros ${ }^{2}$, Gustavo José de Araújo Aguiar ${ }^{2}$, Armando Dias Duarte ${ }^{3}$ e Gilson Lima da Silva4}

${ }^{1}$ Universidade Federal de Pernambuco. Núcleo de Tecnologia. Campus Caruaru. Av. Marielle Franco, S/№, km 59. Nova Caruaru. Caruaru-PE, Brasil (CEP 55014900).*E-mail: floro@hotmail.com.br.

${ }^{2}$ Universidade Federal de Pernambuco. Programa de Pós-Graduação em Engenharia Civil e Ambiental. Campus Caruaru. Av. Marielle Franco, S/№, km 59. Nova Caruaru. Caruaru-PE, Brasil (CEP 55014-900).

${ }^{3}$ Universidade Federal de Pernambuco. Programa de Pós-Graduação em Engenharia Civil. Campus Recife. Av. Prof. Moraes Rego, 1235. Cidade Universitária. Recife-PE, Brasil (CEP 50670-901).

${ }^{4}$ Universidade Federal de Pernambuco. Departamento de Recursos Hídricos. Programa de Pós-Graduação em Engenharia Civil e Ambiental. Campus Caruaru. Av. Marielle Franco, S/№, km 59. Nova Caruaru. Caruaru-PE, Brasil (CEP 55014-900).

Resumo. Compreender o saneamento ambiental é de suma importância para a elaboração de ações públicas, que visam à melhoria das questões insalubres das comunidades.0 manejo das águas pluviais em conjunto com as águas servidas, apresenta-se como um desafio na gestão dos municípios brasileiros, através das vazões domésticas, industriais e parasitárias. 0 presente estudo tem como objetivo compreender a influência e os impactos das vazões parasitárias à rede de esgoto de um município de médio porte, avaliadas as preciptações em um período de cinquenta anos. Para o desenvolvimento da pesquisa,foram utilizadosdados hidrológicos e de manutenção da rede, no qual foram obtidos coeficientes de correlação entre as variáveis do estudo. Foi observado aumento dos serviços de manutenção durante o período chuvoso, através da contribuição de ligações irregulares entre outros fatores físicos.

Palavras-chave: Recursos hídricos; Salubridade; Drenagem urbana.

Abstract. Assessment of the impacts of rainfall on the maintenance of the sewage network in a municipality in the Agreste Region of Pernambucano. Understanding

Recebido

$18 / 11 / 2021$

Aceito

$29 / 12 / 2021$

Disponível on line $31 / 01 / 2022$

Publicado $30 / 04 / 2022$

Acesso aberto

ISSN 2359-1412/RBGAS-2021-0148/2022/9/21/1/3

Rev. Bras. Gest. Amb. Sustent.

http://revista.ecogestaobrasil.net 
environmental sanitation is of paramount importance for the development of public actions, aimed at improving unhealthy issues in communities. The management of rainwater together with wastewater is a challenge in the management of Brazilian municipalities, through domestic, industrial and parasitic flows. The present study aims to understand the influence and impacts of parasitic discharges to the sewage network of a medium-sized municipality, evaluating the precipitations over a period of fifty years. For the development of the research, hydrological and network maintenance data were used, in which correlation coefficients between the study variables were obtained. An increase in maintenance services was observed during the rainy season, through the contribution of irregular connections and other physical factors.

Keywords: Water resources; Health; Urban drainage.

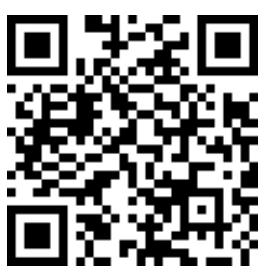

\author{
ORCID \\ (1) $0000-0002-7586-2997$ \\ José Floro de Arruda \\ Neto \\ (1) 0000-0001-7112-5984 \\ Iálysson da Silva \\ Medeiros \\ (1) 0000-0002-4727-2981 \\ Gustavo José de Araújo \\ Aguiar \\ (D) 0000-0003-2571-7705 \\ Armando Dias Duarte \\ (1) 0000-0003-2484-3590 \\ Gilson Lima da Silva
}

\section{Introdução}

0 conceito de saneamento ambiental de acordo com Kronemberger et al. (2011) e Nugem et al. (2021), pode ser entendido como o conjunto de ações integradas para proteger e melhorar as condições ambientais e humanas abrangendo a coleta e disposição sanitária de resíduos sólidos e líquidos, acesso ao abastecimento de água potável, ocupação e disciplina no uso territorial, intervenções para proteção e melhoria da qualidade de vida, controle de vetores e doenças transmissíveis e drenagem urbana. Entre as intervenções públicas destacam-se os serviços de infraestrutura, entre eles os níveis de esgotamento sanitário ofertado e qualidade deste, são diretamente mensurados para a obtenção do Índice de Desenvolvimento Humano (IDH) (Guimarães et al., 2005). Vale destacar a que há uma diferença entre esgotamento sanitário e tratamento de esgoto, em síntese, o primeiro trata da condução do efluente entre a geração e o corpo receptor, enquanto o segundo adiciona o tratamento do efluente ao conceito anterior (Rebelo et al., 2012).

O sistema de esgotamento sanitário é fundamental para a preservação da saúde populacional e preservação do meio físico ambiental, sua implantação, operação e manutenção das redes estão intimamente ligados a seguridade de alguns dos direitos fundamentais, previstos no art. 5o , da Constituição Federal (Pereira, 2003). Desde a antiguidade, medidas de tratamento de água para consumo e separação de águas servidas são vistas como forma de desenvolvimento e preocupação da humanidade, então, o lançamento de dejetos em vias e corpos hídricos foi substituído por soluções individuais de tratamento e posteriormente por sistemas coletivos maiscomplexos.

Inicialmente os sistemas de esgotamento europeus e americanos foram concebidos para coletar e transportar águas de chuva, reduzindo alagamentos urbanos. Em 1815, Londres autoriza a utilização destes sistemas para lançamento de esgoto doméstico, passando a obrigatória apenas em 1847, essa transiçãofez surgir um novo problema, a transferênciadainsalubridade das casas para o rio Tâmisa, que em 1850 já apresentava 
considerável índice de poluição facilitando a propagação da cólera (Vibrio Cholerae) (Azevedo Netto et al., 1983).

De acordo com Dias (2003), a evolução do esgotamento sanitário pode ser avaliada através da seguinte ótica: a) Higienista: no qual os profissionais de saúde defendiam a eliminação de áreas alagadas e os sistemas individuais passaram a ser substituídos por sistemas coletivos canalizados; b) Racionalização e normatização dos cálculos hidrológicos: caracterizada pela adoção de normas para definir os ciclos hidrológicos, com o principal objetivo em dimensionar obras hidráulicas, foi diretamente motivada pelas epidemias de cólera na Europa do século XIX; c) Científica e ambiental do ciclo hidrológico urbano: na primeira fase, a principal preocupação era afastar as águas servidas e controlar os eventos de enchentes, sem a devida preocupação ambiental e bastante similar a fase anterior.

No Brasil é utilizado o sistema separador absoluto para efluentes de esgotamento, havendo dois sistemas, o sistema sanitário e pluvial (Brasil, 2019). 0 sistema de drenagem pluvial é composto por canais naturais e artificiais, condutos e equipamentos com o objetivo de reduzir os impactos das precipitações pluviométricas como enchentes, deslizamentos, assoreamentos, entre outros (Brasil, 2019). Já o sistema de esgotamento sanitário é composto pelo conjunto de condutos e equipamentos destinados a captação, transporte, tratamento e destinação final de águas servidas de origem industrial e residencial. Na literatura é comum dividir o esgoto em industrial e doméstico, sendo possível observar a nomenclatura de esgoto pluvial para o efluente das chuvas, no presente trabalho, será adotada a nomenclatura drenagem urbana para estas vazões, a forma usual (Brasil, 2019).

Os efluentes conduzidos no sistema de esgotamento sanitário são compostos por águas servidas domésticas, industriais e de infiltração em sistemas que podem ser classificados em individuais ou coletivos(Von Sperling, 2005; Brasil, 2019). 0 sistema individual é constituído por fossa séptica e dispositivo de infiltração, podendo ser a fossa do tipo sumidouro ou vala de infiltração superficial descrito pela norma ABNT NBR 13.969:1997 (ABNT, 1997). No Brasil e em países em desenvolvimento, a utilização de sistemas individuais continua sendo a solução encontrada por parte da população como tentativa de suprir a insuficiência de investimento público em saneamento. Em diversas ocasiões este descompasso entre a necessidade e o recurso investido é reflexo do acelerado crescimento populacional e da dificuldade do poder público de criar estratégias para acompanhar este crescimento (Jordão e Pessôa, 2011). Por outro lado os sistemas coletivos podem se subdividir em sistema de esgotamento unitário ou combinado, sistema de esgotamento separador parcial e sistema de esgotamento separador absoluto, sendo este o mais utilizado atualmente, inclusive sendo o adotado no Brasil (Tsutiya e Além Sobrinho, 2011; Freire, 2014).

0 regime de chuvas é o principal fator para definição do sistema de separação. Em regiões tropicais as precipitações são intensas e de baixa frequência inviabilizando os sistemas unitários onde seriam necessárias grandes capacidades de escoamento para épocas chuvosas, ficando subutilizados em épocas de menor precipitação. Por outro lado, em regiões de clima temperado as chuvas são distribuídas com maior uniformidade consequentemente apresentando menores intensidades e maior regularidade das frequências, somado a isto as precipitações são geralmente inferiores a 1/3 das de países de clima tropical possibilitando a escolha dos sistemas unitários (Volschan et al., 2009; Brasil, 2019).

No sistema unitário, os efluentes provenientes do esgoto doméstico, infiltração, pluvial e eventuais despejos industriais são conduzidos em um único condutor, demandando diâmetros elevados de canalização visto que o dimensionamento do conduto é baseado nas precipitações máximas (Brasil, 2019). No Brasil o modelo não é permitido,

Rev. Bras. Gest. Amb. Sustent., 2021, vol. 9, n. 21, p. 3-19. 
entretanto países da Europa, Ásia e em cidades da América do Norte, onde os sistemas de esgotamento sanitários foram implantados antes do surgimento de sistemas mais modernos, o sistema unitário é facilmente encontrado (Tsutiya e Bueno, 2004; Brasil, 2019).

O modelo separador parcial apresenta separação entre águas de esgoto e parte das águas de chuva, sendo admitidos os escoamentos de telhados, calçadas, etc. No Brasil é possível encontrar este sistema em cidades como Joinville e Porto alegre, havendo o compromisso de separação futura dos efluentes ao longo do processo (Tsutiya e Bueno, 2004; Tsutiya \& Além Sobrinho, 2011; Brasil, 2019). Em diversas situações os sistemas são planejados como separadores absolutos e de forma irregular são utilizados como separadores parciais. Entre as possíveis causas destacam-se a falta de fiscalização das obras de implantação e de operação das redes, questões ligadas também à educação sanitária dos usuários (Dias e Rosso, 2011). Embora este formato pareça mitigar os problemas de drenagem urbana pode trazer diversas consequências ao meio ambiente, aumentando o risco de contaminação nas bacias onde está situado (Festi, 2006).

As vazões do sistema de esgotamento sanitário são formadas pelas vazões industriais, domésticas, vazão de infiltração do solo e contribuição pluvial parasitária conforme descrito pela ABNT NBR 9648:1986 (ABNT, 1986). Apesar da legislação vigente, o sistema separador absoluto em muitas cidades brasileiras não é de fato observado, funcionando como separador parcial, onde observa-se grande aumento de vazões de esgotamentoem períodos chuvosos nas estações de tratamento, comprometendo significativamente o sistema pelo aumento de vazão e arrasto de sólidos (Tsutiya et al., 2003; Pires, 2018).

A vazão parasitária é formada, em geral, pela injeção no sistema, de forma acidental ou clandestina, de contribuição pluviométrica, a qual não deveria ter acesso a este. 0 acesso acidental ocorre através de falhas na estanqueidade do sistema ou de forma clandestina através de ligações irregulares, esta situação teoricamente ocorre devido à falta ou insuficiência de controle às ligações irregulares, normalmente em redes antigas, por questões culturais, entre outros, este tipo de contribuição é ligada ao regime pluviométrico local, consequentemente sazonal e cíclica (Rosso et al., 2011).

0 conceito de saneamento ambiental evoluiu bastante, passando do tratamento de esgoto para o conjunto de ações que visam aumentar a salubridade do meio natural em decorrência de ações antrópicas (Guimarães et al., 2005). Atualmente o saneamento ambiental é considerado uma atividade estratégica para melhoria da saúde pública, redução da proliferação de doenças e consequente redução dos gastos hospitalares.

No Brasil, de acordo com Vaz (2015), alguns estudos apontam carência neste setor, em locais onde residem familias de baixa renda e escolaridade, as quais não escolhem morar nestas áreas, ausentes de saneamento básico, e sim dependem destas para sua sobrevivência. Além disso, esses locais apresentam esta condição por por ausência de investimentos públicos em saneamento e equipamentos urbanos e não por ação direta de seus habitantes. Consequentemente a Lei no 14.026/2020, que atualiza o marco legal do saneamento básico, torna visível a preocupação com o estabelecimento de metas para universalização do saneamento público e viabilidade econômica da prestação destes serviços (Brasil, 2020).

A rede de esgotamento no Brasil ainda é considerada insuficiente para propiciar a universalização que o país necessita e garantir segurança sanitária à população. Ademais, conhecer os fatores que impactam as redes existentes é fundamental para o planejamento de intervenções para sua manutenção e expansão. Neste sentido, o objetivo deste estudo é entender os motivos que levam ao aumento de problemas de obstrução da rede de esgotamento sanitário doméstico em períodos chuvosos e os diversos impactos causados 
através da identificação de correlações entre os dados hidrológicos e a manutenção da rede de esgotamento.

\section{Metodologia}

O local de desenvolvimento do estudo foi Município de Caruaru, no Estado de Pernambuco, situado na Mesorregião do Agreste e Microrregião do Vale do Ipojuca, a cerca de $130 \mathrm{~km}$ da capital, com área de $923,15 \mathrm{~km}^{2}$. Possui população total estimada em 365.278 habitantes (IBGE, 2020), destes cerca de 89\% residem em área urbana. 0 Município de Caruaru possui cerca de 114.621 domicílios sendo a taxa de ocupação 3,26 hab./domicílio, dos quais $81,3 \%$ são atendidos por redes de esgotamento sanitário (IBGE, 2010), sendo ela de aproximadamente $391 \mathrm{~km}$ (IBGE, 2017).

Para a realização do estudo foram utilizados dois conjuntos de dados, os de manutenção da rede de esgotamento sanitário e os hidrológicos da localidade.

\section{Dados de manutenção da rede e hidrológicos}

Os dados referentes à manutenção da rede foram obtidos, em conjunto à Secretaria de Infraestrutura Urbana e Obras de Caruaru (SIURB), através da conferência de ordens de serviço, boletins de medição e notas fiscais das atividades pertinentes ao estudo realizados nos anos de 2018, 2019 e 2020, referentes à desobstrução de tubulação de ligação predial, coletores secundários, coletores principais e coletores tronco, limpeza de terminais e caixas de passagem, reformas de caixas de passagem, construção de caixas de passagem e fornecimento de tubos, os quais serão discutidos a seguir (SIURB, 2021).

A localidade apresenta rede de esgotamento de responsabilidade mista, onde a maior parte operada pela Companhia Pernambucana de Saneamento (COMPESA) e parte restante pela Prefeitura Municipal. As redes municipais foram construídas pela prefeitura e ou população, em sua maioria antigas e que não foram absorvidas pela referida concessionária.

Em relação as redes, é possível dividi-las em três momentos históricos, redes antigas construídas sem observância de normatização, dispondo de galerias em alvenaria de pedra ou blocos cerâmicos e tubos cerâmicos presentes em bairros antigos, em maior parte situadas na região central da cidade; redes jovens normatizadas constituídas de coletores em concreto e PVC e ligações em PVC; redes novas caracterizadas por expansões e reformas das redes anteriores.

O município realiza a ampliação, manutenção e monitoramento de parte da rede de esgotos, que não foi integrada à rede COMPESA, bem como das redes de drenagem existentes na localidade (SIURB, 2021).

A secretaria divide a cidade em 10 lotes geográficos compostos por todos os bairros da zona urbana e quatro distritos em sua área rural, as demandas de manutenção da rede de esgoto são obtidas através de monitoramento permanente das equipes e solicitações dos usuários da rede através de visita ao órgão ou através de canais de atendimento da prefeitura (SIURB, 2021).

A Gerência de manutenção utiliza tubos de PVC liso, PVC corrugado, concreto e concreto armado com diâmetros no intervalo de 150,1000 mm, instalados em redes de esgotamento e drenagem pluvial, definidos a partir da normatização vigente (SIURB, 2021).

Foram construídas e organizadas tabelas eletrônicas, dividindo as ações mensalmente e por serviço, sendo possível calcular as médias mensais de execução de manutenção para cada um deles. Os dados das precipitações pluviométricas do Município de Caruaru foram avaliados e um período de 55 anos obtidos através do Instituto de Tecnologia de Pernambuco (ITEP) e Agência Nacional de Águas (ANA), compreendidos entre 1965 a 2020. 
Identificou-se a necessidade de preencher as falhas utilizando os métodos de ponderação regional e regressão linear múltipla similar ao utilizado por Diaz et al. (2018) e descrito por Mello et al. (2017).

Foram determinados diversos parâmetros estatísticos: médias, mínimos, máximos, modas, medianas, curtoses, assimetrias, desvios padrão, absoluto e médio, coeficiente de variância e variância, paracompreender o comportamento hidrológico do município e identificar os períodos de maior incidência de chuvas e a média histórica mensal no município. Foram realizados os testes de correlação entre as médias mensais dos serviços de manutenção da rede de esgoto, agrupadas por anos, e as médias históricas mensais de precipitação para consequentemente avaliação de causalidade entre ambas (Coutinho et al., 2016).

\section{Resultados e discussão}

A partir das informações obtidas, foi possível identificar os períodos de maior incidência de chuvas e a média histórica mensal no município, conforme indica a Tabela 1.

Tabela1. Precipitações médias mensais históricas (1965-2020).

\begin{tabular}{|c|c|c|c|c|c|c|c|c|c|c|c|c|}
\hline & JAN & FEV & MAR & ABR & MAIO & JUN & JUL & AGO & SET & OUT & NOV & DEZ \\
\hline $\mathbf{( m m )}$ & 44,2 & 58,1 & 80,7 & 87,9 & 83,3 & 90,9 & 98,1 & 50,0 & 25,5 & 13,8 & 12,3 & 22,2 \\
\hline Total & 667,02 & - & - & - & - & - & - & - & - & - & - & - \\
\hline
\end{tabular}

Fonte: Brasil (2021).

As chuvas totais observadas têm valores abaixo dos $1.000 \mathrm{~mm} / \mathrm{ano}$, sendo a única exceção os anos de 2004 e 2011 (Figura 1).

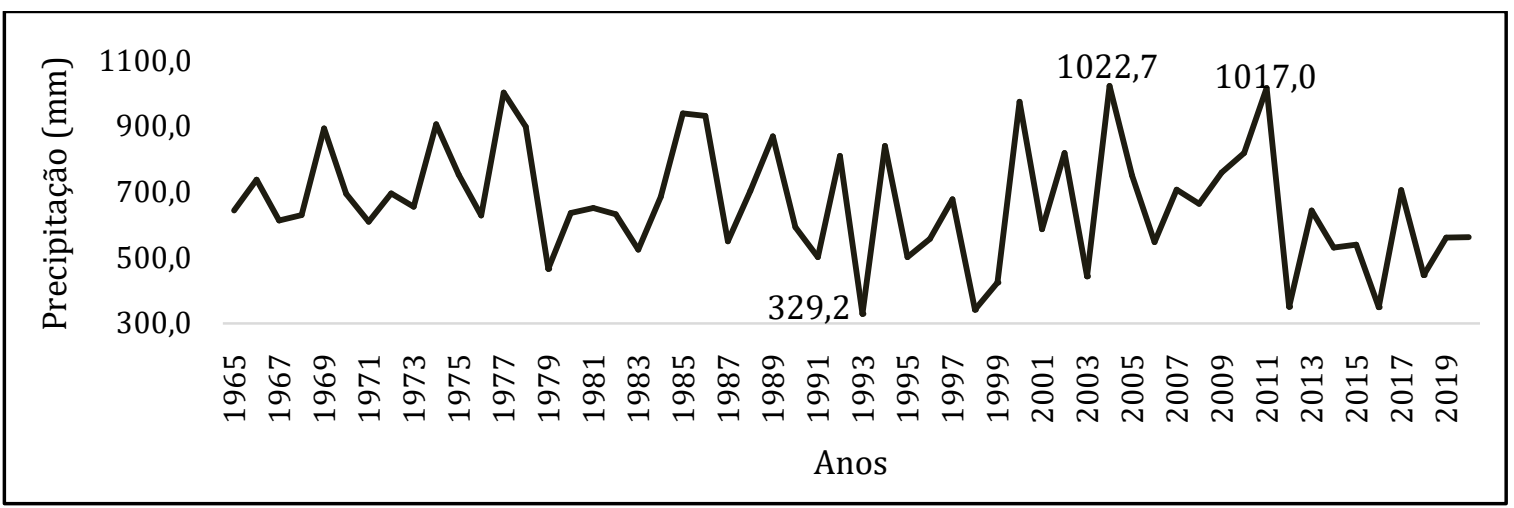

Figura 1.Precipitações acumuladas anuais (1965-2020). Fonte: Brasil (2021).

A irregularidade das chuvas na região é muito intensa, onde a precipitação anual média acumulada $667,02 \mathrm{~mm}$, cerca de $30 \%$ das chuvas são de grande intensidade. A taxa de evapotranspiração é superior as taxas de precipitação, sendo os meses de agosto a 
dezembro considerados críticos, quanto ao déficit hídrico (Santos e Montenegro, 2012; Arruda Neto et al., 2019).

Em relação aos dados de precipitação observou-se que as chuvas totais, apresentaram uma variação entre $329,2 \mathrm{~mm}$, em 1993, a $1.022,7 \mathrm{~mm}$, no ano de 2004 (Figura 1), no qual demonstra um ano marcado por estiagem intensa e outro por enchentes na região confirmado por Arruda Neto et al. (2018). Foi observado uma dispersão entre as médias mensais e os valores de máxima históricos, representando riscos de alagamentos e enchentes no primeiro semestre (Figura 2).

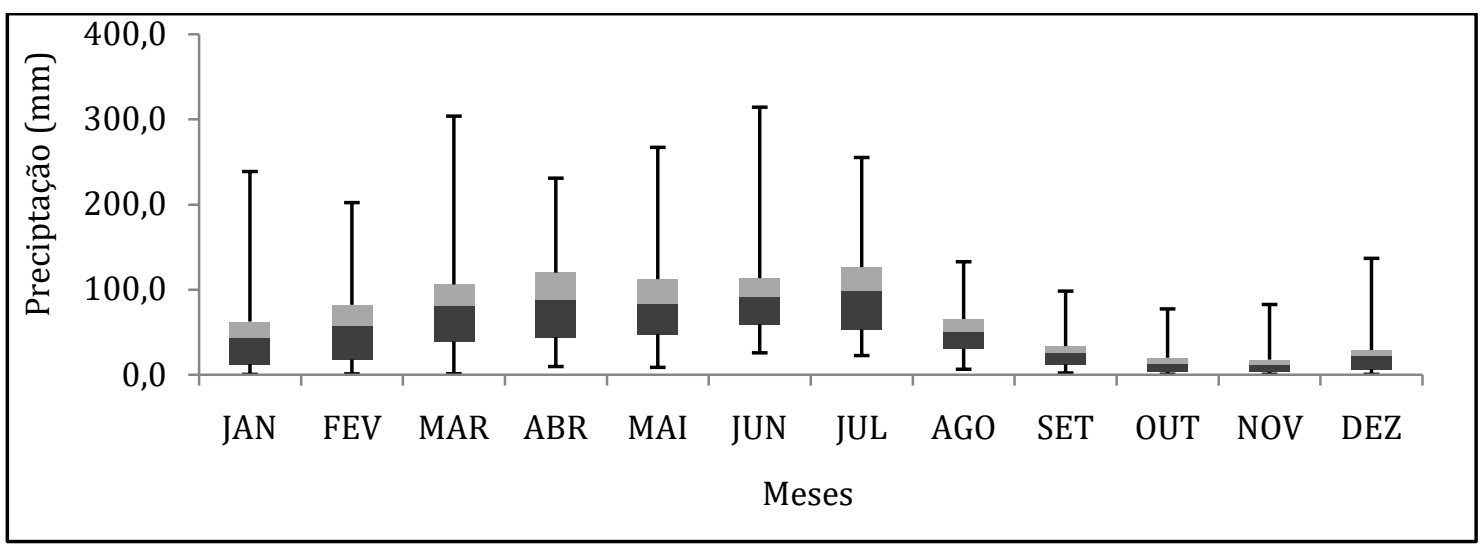

Figura 2. Precipitações médias mensais (1965-2015). Fonte: Brasil (2021).

Também é possível observar na Figura 2 que no intervalo avaliado as precipitações com médias entre 80 e $100 \mathrm{~mm}$ ocorreram entre março e julho, sendo o segundo semestre marcado por precipitações médias abaixo de $60 \mathrm{~mm}$, onde os últimos quatro meses apresentam médias inferiores a $30 \mathrm{~mm}$ de precipitação acumulada, confirmado por Silva et al. (2010) e Arruda et. al (2018). A partir da observação das medianas (Figura 3), foram identificados valores de 74,2 mm, em 2011, e 13,3 mm, em 2015, caracterizando o regime semiárido, ratificado pelos estudos de Andrade et al. (2018) e Arruda Neto et al. (2018).

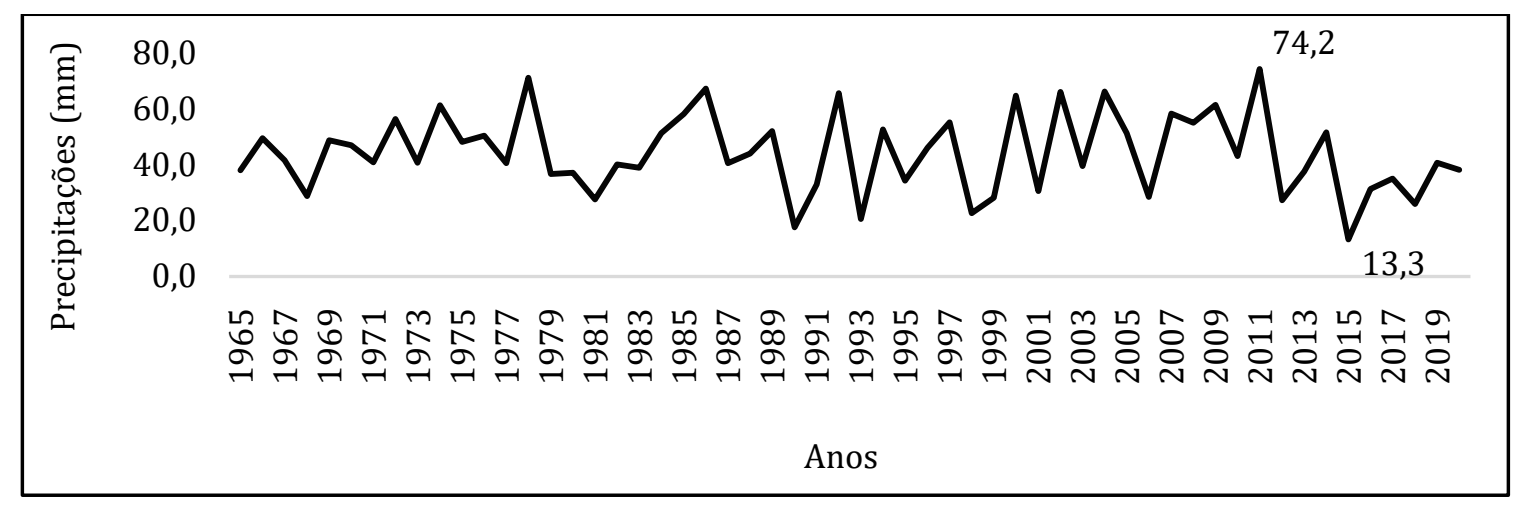

Figura 3. Medianas mensais das precipitações (1965-2020). Fonte: Brasil (2021). 
Quanto ao total mensal do serviço de desobstrução, mecânica, manual e por jateamento (Tabela 2), observa-se que março de 2020 apresenta a maior quantidade de desobstrução da rede $(1.857 \mathrm{~m})$, enquanto agosto de 2020 apresenta o menor valor $(0 \mathrm{~m})$. Observa-se ainda, que em agosto não foram realizadas manutenções na rede por ocasião do fim dos contratos entre as empresas licitadas e a SIURB, sendo ativados os serviços no mês de setembro através de novos contratos.

Tabela 2. Desobstrução de tubulação de esgotamento sanitário (m).

\begin{tabular}{|c|c|c|c|c|c|c|c|c|c|c|c|c|}
\hline & JAN & FEV & MAR & ABR & MAI & JUN & JUL & AGO & SET & OUT & NOV & DEZ \\
\hline $\begin{array}{l}\infty \\
\stackrel{\sim}{ٍ} \\
\stackrel{N}{2}\end{array}$ & 1169,5 & 797 & 1254,6 & 1249,5 & 1296,3 & 1292 & 1129,5 & 539 & 528 & 482 & 519 & 534 \\
\hline 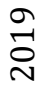 & 1160 & 1014 & 1857 & 1295 & 1198 & 1164 & 1002 & 561 & 470 & 526 & 329 & 447 \\
\hline 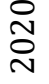 & 1386 & 999 & 1070 & 1071 & 957 & 1192 & 954 & 0 & 921 & 567 & 464 & 454 \\
\hline
\end{tabular}

Os serviços executados de limpezas de caixas e terminais de limpeza da rede abordada durante a manutenção de desobstrução, apresentaram o valor máximo em janeiro de 2018 (246 unidades) e mínimo em agosto de 2020 (0 unidades), conforme Tabela 3.

Tabela 3. Limpeza de terminais e caixas de passagem (un.).

\begin{tabular}{|c|c|c|c|c|c|c|c|c|c|c|c|c|}
\hline & JAN & FEV & MAR & ABR & MAI & JUN & JUL & AGO & SET & OUT & NOV & DEZ \\
\hline 2018 & 246 & 208 & 197 & 213 & 162 & 102 & 83 & 113 & 99 & 66 & 89 & 114 \\
\hline 2019 & 156 & 101 & 196 & 179 & 207 & 154 & 210 & 212 & 102 & 118 & 132 & 121 \\
\hline 2020 & 177 & 98 & 127 & 155 & 103 & 121 & 120 & 0 & 86 & 90 & 30 & 25 \\
\hline
\end{tabular}

Os totais mensais de reformas realizadas em caixas de passagem durante os serviços de manutenção, apresentaaram valor máximo em abril de 2018 (61 unidades) e mínimo em agosto de 2020 (0 unidades), conforme Tabela 4.

Tabela 4. Reformas de Caixas de Passagem (un.).

\begin{tabular}{|c|c|c|c|c|c|c|c|c|c|c|c|c|}
\hline & JAN & FEV & MAR & ABR & MAI & JUN & JUL & AGO & SET & OUT & NOV & DEZ \\
\hline 2018 & 39 & 35 & 46 & 61 & 35 & 19 & 13 & 11 & 9 & 5 & 11 & 9 \\
\hline 2019 & 23 & 18 & 17 & 18 & 20 & 9 & 6 & 9 & 13 & 11 & 7 & 5 \\
\hline 2020 & 8 & 3 & 4 & 7 & 5 & 3 & 7 & 0 & 4 & 13 & 4 & 2 \\
\hline
\end{tabular}

Os valores em metros de fornecimento de tubos, de diâmetros nominais e materiais variados (Tabela 5), utilizados na manutenção da rede de esgotamento sanitário, apresentaram o valor máximo em julho de 2019 (814 m) e mínimo em agosto de 2020 $(0 \mathrm{~m})$. 
Tabela 5. Fornecimento de tubos para rede de esgoto (m).

\begin{tabular}{|c|c|c|c|c|c|c|c|c|c|c|c|c|}
\hline & JAN & FEV & MAR & ABR & MAI & JUN & JUL & AGO & SET & OUT & NOV & DEZ \\
\hline 2018 & 801,5 & 327 & 374 & 336 & 450,9 & 141 & 350 & 308 & 271,2 & 434 & 398 & 272 \\
\hline 2019 & 468 & 322 & 310 & 472 & 535 & 337 & 814 & 409 & 412 & 274 & 148 & 304 \\
\hline 2020 & 279 & 484 & 226 & 229 & 393 & 370 & 452,5 & 0 & 371 & 228 & 99 & 68 \\
\hline
\end{tabular}

Os valores totais mensais relativos à construção de caixas de passagem (Tabela 6), durante a abordagem de manutenção da rede, o valor máximo encontrado foi em fevereiro de 2020 (63 unidades) e mínimo em agosto de 2020 (0 unidades). A construção de novas caixas ocorre quando da necessidade técnicas ou em substituição a caixas existentes que não apresentam possibilidade de reforma.

Tabela 6. Construção de Caixas de passagem (un.).

\begin{tabular}{|c|c|c|c|c|c|c|c|c|c|c|c|c|}
\hline & JAN & FEV & MAR & ABR & MAI & JUN & JUL & AGO & SET & OUT & NOV & DEZ \\
\hline 2018 & 50 & 56 & 58 & 32 & 19 & 20 & 33 & 29 & 35 & 27 & 34 & 22 \\
\hline 2019 & 20 & 16 & 31 & 15 & 16 & 15 & 51 & 29 & 36 & 39 & 30 & 11 \\
\hline 2020 & 29 & 63 & 18 & 19 & 10 & 21 & 18 & 0 & 7 & 13 & 6 & 4 \\
\hline
\end{tabular}

Em relação aos serviços de manutençãoobserva-se que em março de 2019 ocorreu o maior índice de desobstrução de rede $(1857 \mathrm{~m})$, conforme demonstrado na Tabela 2, superior à média do período avaliado $(884,67 \mathrm{~m})$, entretanto o mês com a maior média de desobstrução no estudo é março (1393,87 m), conforme Tabela 7 e Figura 4.

Tabela 7. Média de desobstrução mensal (m/mês) (2018-2020).

\begin{tabular}{|c|c|c|c|c|c|c|c|c|c|c|c|}
\hline JAN & FEV & MAR & ABR & MAI & JUN & JUL & AGO & SET & OUT & NOV & DEZ \\
\hline 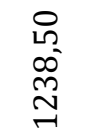 & $\begin{array}{l}\hat{\sigma} \\
\tilde{o}^{-} \\
\text {ă }\end{array}$ & $\begin{array}{l}\hat{\infty} \\
\text { ñ } \\
\tilde{m}\end{array}$ & $\begin{array}{l}\text { 고 } \\
\text { ஸे } \\
\stackrel{+}{\sim}\end{array}$ & 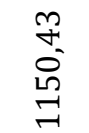 & 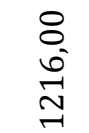 & 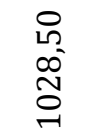 & $\begin{array}{l}\hat{b} \\
\delta^{-} \\
e^{2}\end{array}$ & $\begin{array}{l}\hat{0} \\
\hat{\tilde{\sigma}}\end{array}$ & $\begin{array}{l}8 \\
\text { ஸे } \\
\text { ஸै }\end{array}$ & $\begin{array}{l}m \\
\stackrel{m}{~} \\
\stackrel{m}{+}\end{array}$ & $\begin{array}{l}m \\
m \\
\infty^{0} \\
\stackrel{+}{f}\end{array}$ \\
\hline
\end{tabular}

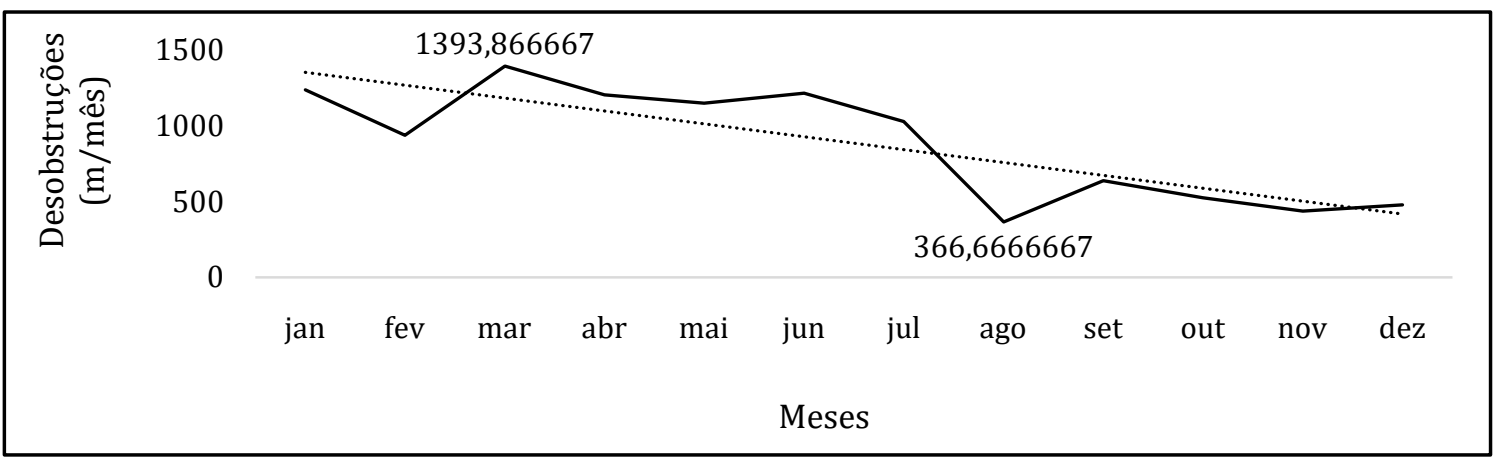

Figura 4.Média de desobstrução mensal (m/mês) (2018-2020). 
0 mês de março tem relevância importante para o estudo por ser o mês onde se iniciam as médias chuvosas acima de $80 \mathrm{~mm}$ e com segunda maior chuva máxima mensal acumulada (303,6 mm), conforme observado na Figura 2, perdendo neste quesito apenas para junho $(314,1 \mathrm{~mm})$. É possível inferir que o início das chuvas fortes no período avaliado pode ter levado ao aumento dos serviços de desobstrução, conforme observado nos estudos similares de Vieira et al. (2015), Paiva et al. (2018) e Fornari Filho et al. (2019). Observa-se forte relação estatística entre os serviços de desobstrução e as médias chuvosas através do método de correlação conforme Tabela 8, ratificado pelos estudos de Festi (2006) e Fornari Filho et al. (2019).

Tabela 8. Correlação de Pearson (r) entre as precipitações médias mensais e os serviços de desobstrução.

\begin{tabular}{|l|c|}
\hline Ano & Coeficiente de correlação (r) \\
\hline 2018 & 0,875659797 \\
\hline 2019 & 0,785315245 \\
\hline 2020 & 0,51722516 \\
\hline $2018-2020$ & 0,719095148 \\
\hline
\end{tabular}

A redução de causa e efeito no ano de 2020 pode ser justificada pela descontinuidade dos serviços no mês de agosto, entretanto é possível observar no período global (2018-2020) coeficiente de correlação de 0,7190, considerada alta de acordo com Watanabe et al. (2009).

Os serviços de limpeza de terminais apresentaram redução nos índices de correlação à média de precipitação em relação aos de desobstrução, é possível inferir que a redução da correlação observada na Tabela 9, deve-se ao fato da rede avaliada ser antiga e não apresentar os terminais de limpeza instalados à rede de forma linear (MELO, 2018). Em conversa com a equipe de manutenção da SIURB tal suposição foi confirmada. Existem ruas dos bairros centrais da cidade com terminais com distância de cerca de 5 metros e ruas de bairros mais periféricos com terminais com distância superior a 10 metros, justificando os índices, embora seja possível observar redução acentuada neste tipo de demanda a partir do mês de agosto, conforme observado na Figura 5. Vale acrescentar que existe determinação da Gerência de Manutenção que durante os serviços de desobstrução todas as caixas do trecho abordado devem ser limpas, mesmo que de forma preventiva.

Tabela 9. Correlação de Pearson (r) entre a precipitação e os serviços de limpeza de terminais.

\begin{tabular}{|l|c|}
\hline Ano & Coeficiente de correlação \\
\hline 2018 & 0,332728529 \\
\hline 2019 & 0,785315245 \\
\hline 2020 & 0,51722516 \\
\hline $2018-2020$ & 0,438679998 \\
\hline
\end{tabular}




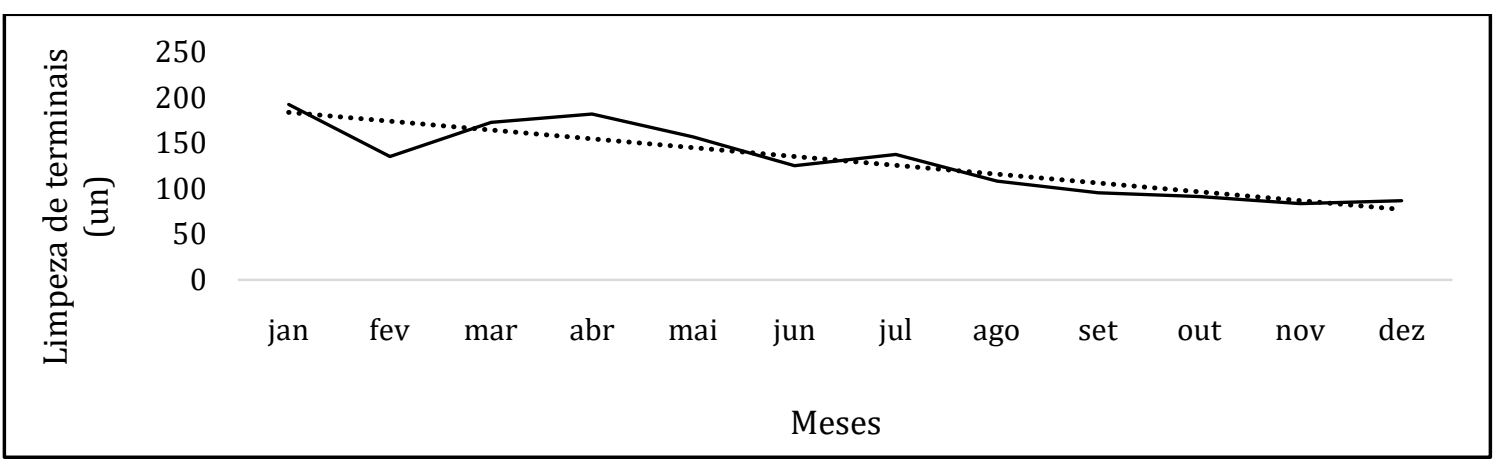

Figura 5. Média de limpeza de terminais (un./mês) (2018-2020).

Situação equivalente ocorre com o serviço de reforma dos terminais conforme Tabela 10, porém com o pico ocorrendo no mês de abril (Figura 6).

Tabela 10. Correlação de Pearson (r) entre a precipitação e os serviços de reforma de terminais.

\begin{tabular}{|l|c|}
\hline Ano & Coeficiente de correlação \\
\hline 2018 & 0,570369623 \\
\hline 2019 & 0,257855883 \\
\hline 2020 & $-0,098783738$ \\
\hline $2018-2020$ & 0,276112056 \\
\hline
\end{tabular}

Em 2020 foi identificada correlação negativa entre as médias mensais de precipitação e os serviços de reforma de terminais, tal situação pode ser explicada como consequência das ações emergenciais da SIURB e da Gestão Municipal de enfrentamento a Pandemia de Covid-19, onde as atividades de manutenção foram focadas em ações de desobstrução da rede e identificação de pontos de reforma para intervenções posteriores, tornando-os irrelevantes ao estudo, conforme descrito por Barbassa e Wright (2005).

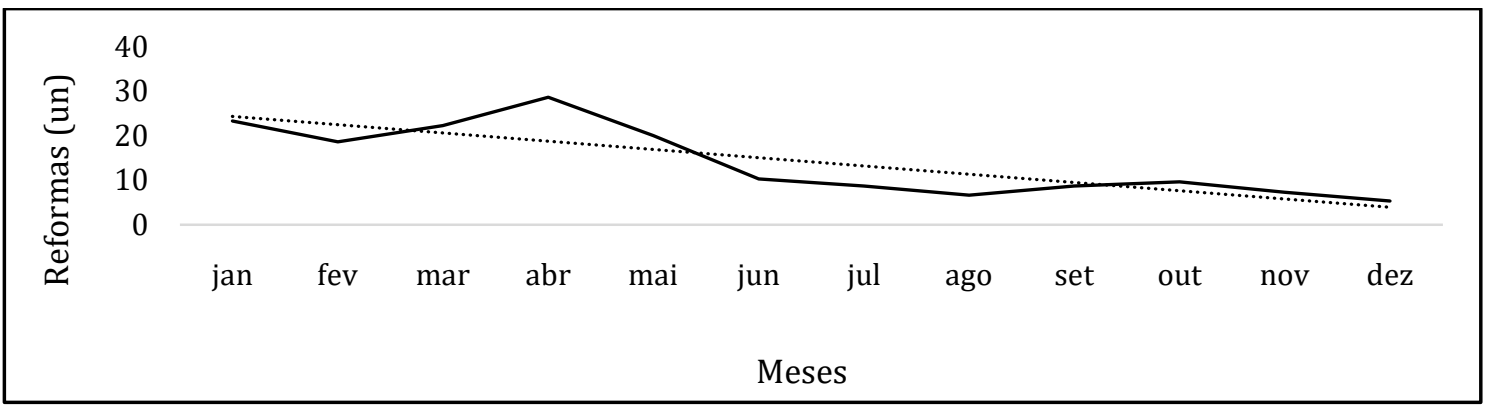

Figura 6. Média de reformas de terminais (un./mês) (2018-2020).

Em relação ao fornecimento de tubos observou-se que em 2018 que a correlação é fraca, aumentando nos anos subsequentes, porém não de forma suficiente para ligar a causa ao efeito conforme Tabela 11. Identifica-se também que em 2018 houve correlação 
negativa sendo possível inferir que em ambas as situações a troca de tubo não se fez necessária em períodos de maiores intensidade de chuva pois a vida útil dos condutores de PVC não é reduzida pelo aumento do fluxo, o que torna a intervenção impertinente para o estudo, conforme preconizado por Barbassa \& Wright (2005).

Tabela 11. Correlação de Pearson (r) entre a precipitação e fornecimento de tubos.

\begin{tabular}{|l|c|}
\hline Ano & Coeficiente de correlação \\
\hline 2018 & $-0,179317331$ \\
\hline 2019 & 0,631739342 \\
\hline 2020 & 0,497430187 \\
\hline $2018-2020$ & 0,299939262 \\
\hline
\end{tabular}

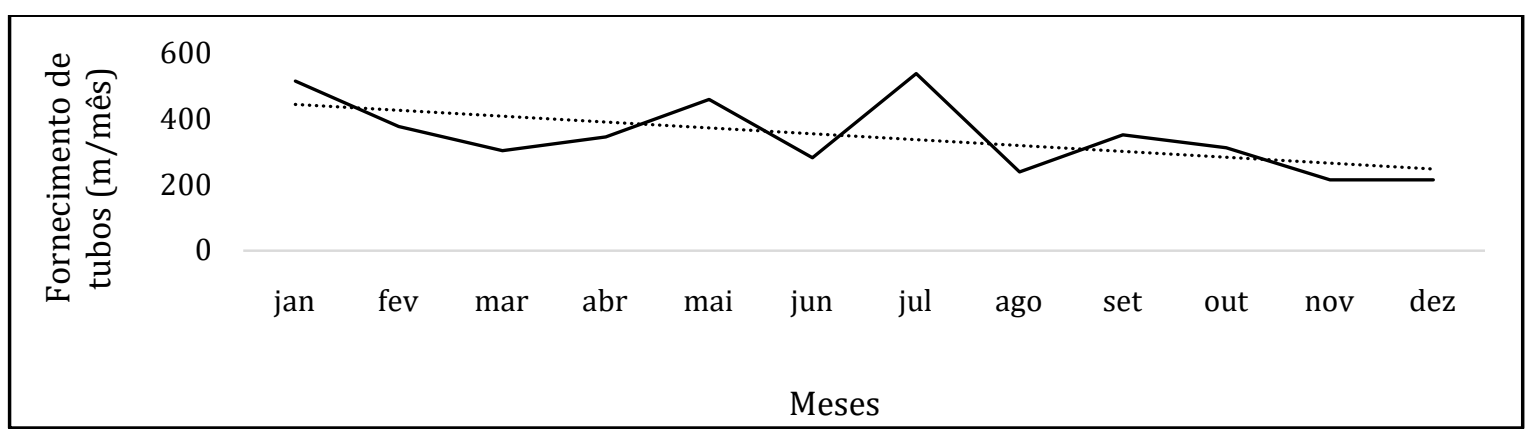

Figura 7. Média de fornecimento de tubos (m/mês) (2018-2020).

Com os dados das médias de fornecimento de tubos novos, é possível observar na Figura 7 que existe uma dispersão, com pontos de máximo nos meses de janeiro, maio e julho, tal situação ocorre, segundo informações levantadas, em função de em janeiro existir forte trabalho preventivo focadono período chuvoso a ser iniciado, seguido de reduções nos fornecimentos no período onde ocorre a maior incidência de demandas, no qual as equipes emergencialmente realizam trabalhos de desobstrução entre março e junho, por serem os meses de chuvas mais intensas, conforme Figura 2. Passado o mês de junho a rede é reformada intensamente em julho e volta ao ritmo normal no segundo semestre, situação equivalente ocorre com a construção de caixas novas conforme observado na Figura 8.

Fator similar observa-se em relação a construção de novas caixas de passagem, conforme Tabela 12, neste ponto é possível observar correlação negativa em 2019 evidenciando-se que as modificações nas médias mensais de precipitação não condicionam a necessidade da construção de novas caixas, tornando o serviço sem relevância para o estudo conforme Barbassa \& Wright (2005).

Tabela 12. Coeficiente de correlação entre a precipitação e construção de caixas.

\begin{tabular}{|l|c|}
\hline Ano & Coeficiente de correlação \\
\hline 2018 & 0,03167211 \\
\hline 2019 & $-0,07454576$ \\
\hline 2020 & 0,28631204 \\
\hline $2018-2020$ & 0,08652673 \\
\hline
\end{tabular}




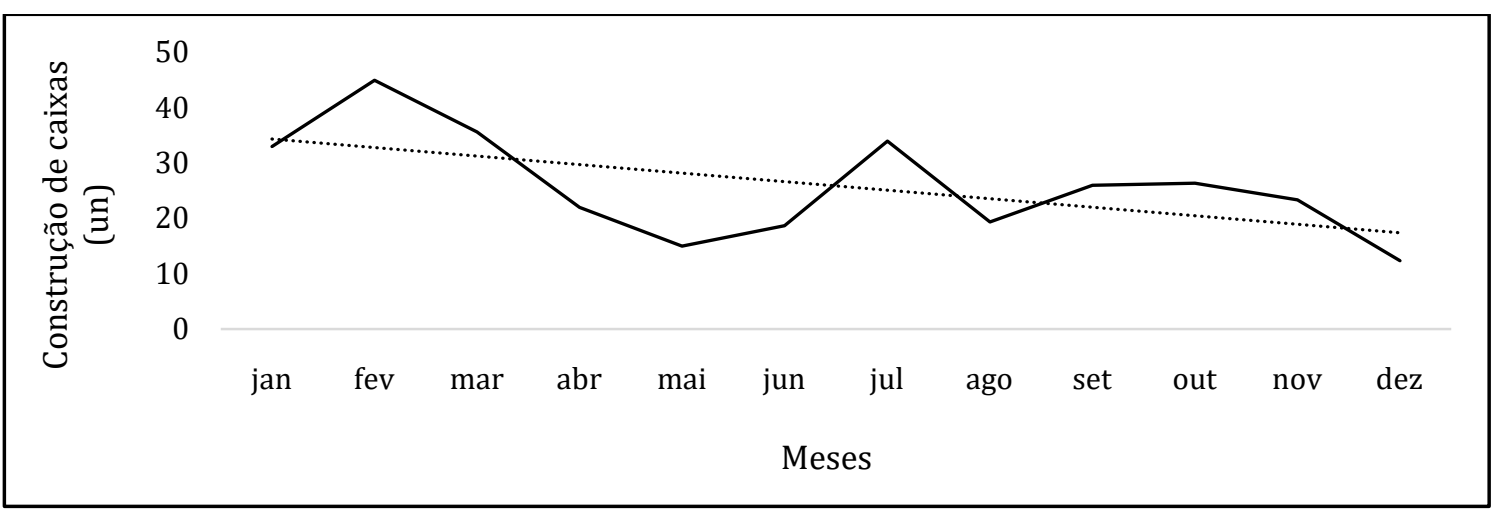

Figura 8. Média de construção de caixas (un./mês) (2018-2020).

Para avaliação de todos os serviços vale destacar a atipicidade do ano 2020, onde o mundo enfrentou a pandemia da Covid-19, não sendo diferente no município de estudo, onde ocorreram-se momentos de fechamento de empresas e quarentena, que podem ter influenciado nos padrões de vida da população e consequentemente no esgotamento sanitário (Almeida et al., 2020).

\section{Conclusões}

A partir dos dados pluviométricos e de serviços de manutenção realizadas na rede de manutenção do município estudado, foi possível realizar estudos estatísticos e constatar a existência de relação entre o período chuvoso e o aumento das obstruções à rede de saneamento. Em todos os casos foi observado redução dos serviços de manutenção em relação ao período onde se concentram as maiores médias pluviométricas, tal fato pode ser explicado pela contribuição parasitária ao sistema, ou seja, ligações irregulares de sistemas de drenagem dos domicílios à rede de esgotamento. Esta contribuição pode levar ao entupimento das redes por diversos fatores, entre eles o arrasto de sólidos como areias e resíduos sólidos, vazões incompatíveis com o dimensionamento da rede, pressurização de condutos que deveriam escoar apenas por gravidade, entre outros.

Tendo em vista a pluviosidade média anual verificada de 667,02 mm/ano, é possível inferir que cerca de 670 litros de água de chuva são adicionados por ano ao sistema de esgotamento sanitário para cada $\mathrm{m}^{2}$ de coberta domiciliar, através de ligação irregular à rede, sendo automaticamente contaminados e sobrecarregando todo o sistema, inclusive parte desta contribuição chegando diretamente ao Rio Ipojuca, que corta o município.

O aumento da demanda de serviços de manutenção de rede no período chuvoso representa aumento dos gastos públicos e consequente destinação de valores que poderiam ser investidos em serviços de infraestrutura e melhoria social, interferindo diretamente na qualidade de vida da população.

Para trabalhos futuros são sugestões utilizar dados de manutenção do sistema gerido pela empresa concessionária de água e esgoto, e verificar se existe compatibilidade entre os resultados, além de incluira quantidade de domicílios ligados irregularmente ao sistema, a fim de estimar o impacto ambiental, social e econômico causado por estas ligações clandestinas 


\section{Agradecimentos}

Os autores agradecem ao Grupo de Gestão Ambiental Avançada (GAMA), da Universidade Federal de Pernambuco (UFPE) e ao Centro Acadêmico do Agreste, Caruaru$\mathrm{PE}$, por todo o apoio e incentivo para o desenvolvimento da pesquisa. 0 presente trabalho foi realizado com apoio da Coordenação de Aperfeiçoamento de Pessoal de Nível Superior - Brasil (CAPES) - Código de Financiamento 001.

\section{Conflito de interesses}

Os autores declaram não haver conflito de interesses.

\section{Referências}

ABNT - Associação Brasileira de Normas Técnicas. ABNT NBR 13.969: Tanques sépticos - Unidades de tratamento complementar e disposição final dos efluentes líquidos Projeto, construção e operação. Rio de Janeiro: ABNT, 1997.

ABNT - Associação Brasileira de Normas Técnicas. ABNT NBR 9.648: Estudo e concepção de sistemas de esgoto sanitário. Rio de Janeiro: ABNT, 1986.

Almeida, C.; Lüchmann, L.; Marteli, C. A pandemia e seus impactos no Brasil. Middle Atlantic Review of Latin American Studies, v. 1, n. 4, p. 20-25, 2020. http://doi.org/10.23870/marlas.313

Andrade, A. R. S.; Godoy Neto, A. H.; Silva, C. A. F.; Andrade, E. K. P.; Santos, V. F.; Silva, T. N. P. Geoestatística aplicada à variabilidade espacial e padrões nas séries temporais da precipitação no agreste pernambucano. Journal of Environmental Analysis and Progress, v. 3, n. 1, p. 126-145, 2018. https://doi.org/10.24221/jeap.3.1.2018.1668.126145

Arruda Neto, J. F.; Duarte, A. D.; Medeiros, I. S.; Aguiar, G. J. A.; Silva, G. L. Adequação de telhados verdes extensivos para a Cidade de Caruaru-PE baseada na média de precipitações chuvosas. In: Silva, H. C. Gestão de recursos hídricos e sustentabilidade 3. 1. ed. Ponta Grossa: Atena, 2019. v. 1. p. 1-8.

Azevedo Netto, J. M.; Botelho, M. H. C.; Garcia, M. A evolução dos sistemas de esgotos. Engenharia Sanitária, v. 22, n. 2, p. 226-228, 1983.

Barbassa, A. P.; Wright, L. Pitfalls of over-calibrating a model of infiltration and inflow. Word Water Congress, Alaska, 2005.

Benchimol, J.; Pereira, L. P. Um Haussmann tropical. Rio de Janeiro: Secretaria Municipal de Cultura, Turismo e Esportes, 1990. (Coleção Biblioteca Carioca, v. 11).

Brasil. Manual de saneamento. 5. ed. Brasília: Funasa, 2019.

Brasil. Lei no 14.026, de 15 de julho de 2020. Atualiza o marco legal do saneamento básico e altera a Lei oㅗ 9.984, de 17 de julho de 2000, para atribuir à Agência Nacional de Águas e Saneamento Básico (ANA) competência para editar normas de referência sobre o serviço de saneamento, a Lei no 10.768 , de 19 de novembro de 2003, para alterar o nome e as atribuições do cargo de Especialista em Recursos Hídricos, a Lei no 11.107, de 6 de abril de 2005, para vedar a prestação por contrato de programa dos serviços públicos de que trata o art. 175 da Constituição Federal, a Lei no 11.445, de 5 de janeiro de 2007, para aprimorar as condições estruturais do saneamento básico no País, a Lei no 12.305, de 2 de agosto de 2010, para tratar dos prazos para a disposição final ambientalmente adequada 
dos rejeitos, a Lei no 13.089, de 12 de janeiro de 2015 (Estatuto da Metrópole), para estender seu âmbito de aplicação às microrregiões, e a Lei no 13.529, de 4 de dezembro de 2017, para autorizar a União a participar de fundo com a finalidade exclusiva de financiar serviços técnicos especializados. Disponível em: <http://www.planalto.gov.br/ccivil_03/ _ato2019-2022/2020/lei/l14026.htm>. Acesso em: 23 fev. 2021.

Brasil. Agência Nacional de Águas (ANA). Séries históricas de precipitações. 2021. Disponível em: <http://www.snirh.gov.br/hidroweb/serieshistoricas>. Acesso em: 14 set. 2021.

Coutinho, E. R.; Silva, R. M.; Delgado, A. R. S. Utilização de técnicas de inteligência computacional na predição de dados meteorológicos. Revista Brasileira de Meteorologia, v. 31, n. 1, p. 24-36, 2016.

Dias, M. S. O. Sistema de esgotamento sanitário no Município Belém: proposta de concepção para universalização do atendimento até 2030. Belém: Universidade Federal do Pará, Belém, 2009. (Dissertação de mestrado).

Dias, A. P.; Rosso, T. C. A. Análise dos elementos atípicos do sistema de esgoto - separador absoluto - na Cidade do Rio de Janeiro. Engevista, v. 13, n. 3, p. 177-192, 2011.

Diaz, C. C. F.; Pereira, J. A. S.; Nobrega, R. S. Comparação de dados estimados pelo método da ponderação regional (PR) e dados estimados pelo TRMM para o preenchimento de falhas de precipitação na Bacia Hidrográfica do Rio Pajeú. Revista Brasileira de Climatologia, v. 22, p. 324-339, 2018.

Festi, A. V. Estimativa da infiltração e do afluxo devidos à precipitação na rede de esgoto sanitário em município de pequeno porte. São Carlos: Universidade Federal de São Carlos, 2006. (Dissertação mestrado).

Freire, J. R. P. Análise do sistema separador absoluto no âmbito da drenagem pluvial da Cidade de Campina Grande: estudo de caso do Canal das Piabas. Campina Grande: Universidade Federal de Campina Grande, 2014. (Dissertação de mestrado).

Fornari Filho, P. F.; Fonseca, F. M. M. L.; Andrade, F. M. Influência das chuvas de verão no SES Costa Norte: comparativo de vazões do efluente da ETE Canasvieiras entre Jan/2017 e Jan/2018. Anais do 30 Congresso Brasileiro de Engenharia Sanitária e Ambiental, ABES, 2019.

Guimarães, R. S.; Jannuzzi, J.; Martino, P. IDH indicadores sintéticos e suas aplicações em políticas públicas: uma análise crítica. Revista Brasileira de Estudos Urbanos e Regionais, v. 7, n. 1, p. 73-90, 2005.

Guimarães, A. J. A.; Carvalho, D. F.; Silva, L. D. B. Saneamento básico. Disponível em: <http://www.ufrrj.br/institutos/it/deng/leonardo/downloads/APOSTILA/Apostila IT 179/Cap 1.pdf>. Acesso em: 23 jul. 2021.

IBGE - Instituto Brasileiro de Geografia e Estatística. Censo 2010. Disponível em: <https://cidades.ibge.gov.br/brasil/pe/caruaru/pesquisa/23/27652?detalhes=true>.

Acesso em: 14 ago. 2021.

IBGE - Instituto Brasileiro de Geografia e Estatística. Pesquisa Nacional de Saneamento Básico. 2017. Disponível em: <https://cidades.ibge.gov.br/brasil/pe/caruaru/pesquisa/ 30/84366>. Acesso em: 14 ago. 2021.

IBGE - Instituto Brasileiro de Geografia e Estatística. Panorama municipal 2021. Disponível em: <https://cidades.ibge.gov.br/brasil/pe/caruaru/panorama>. Acesso em: 14 ago. 2021. 
Jordão, E. P.; Pessôa, C. A. Tratamento de Esgotos Domésticos. 5. ed. Rio de Janeiro: ABES, 2011.

Kronemberger, D. M. P.; Pereira, R. S.; Freitas, E. A. F. Saneamento e meio ambiente: atlas de saneamento. Rio de Janeiro: IBGE, 2011.

Mello, D. E. Análise evolutiva da rede de tratamento de esgoto no Município de Florianópolis, Santa Catarina. Medianeira: Universidade Tecnológica Federal do Paraná, 2018. (Trabalho de conclusão de curso).

Mello, Y. R.; Kohls, W.; Oliveira, T. M. N. Uso de diferentes métodos para o preenchimento de falhas em estações pluviométricas. Boletim de Geografia, v. 35, n. 1, p. 112-121, 2017.

Nugem, R. C.; Bordin, R.; Pedrozzo, E. A.; Rosa, R. S. Abordagem hidrossocial: uma ponte entre o saneamento básico e o saneamento ambiental. In: Oliveira, C. C.; Binkowski, P.; Albrecht, C. A. M.; Silva, J. P. S. Cidades e sustentabilidade: os desafios para conciliar equilíbrio ecológico e bem-estar social. Porto Alegre: UERGS, 2021. p. 63-74.

Paiva, M. A.; Bracarense, D. C.; Sousa, W. S. V. Avaliação do impacto da água de chuva na vazão afluente em uma estação de tratamento de esgoto da Cidade de Palmas (TO). Anais do 29을 Congresso Nacional de Saneamento e Meio Ambiente,São Paulo, 2018.

Pereira, J. A. R. Saneamento ambiental em áreas urbanas. Belém: UFPA, 2003.

Pires, F. M. B. Sistema de esgotamento sanitário: estudo de caso: Maré. Rio de Janeiro: Universidade Federal do Rio de Janeiro, 2018. (Projeto de graduação).

Rebelo, M. M. P. S.; Santos Neto, A. G.; Vasconcelos, S. M.; Ferreira, I. V. L.; Barboza, M. G. Segregação e tratamento de águas cinzas e negras: estudo de caso em uma residência unifamiliar. Anais do XXXIII Congresso Interamericano de Engenharia Sanitária e Ambiental, Salvador, 2012.

Rosso, T. C. A.; Dias, A. P.; Giordano, G. Vulnerabilidade dos sistemas de esgotamento sanitário da Cidade do Rio de Janeiro, RJ, Brasil: relato de um caso de estudo. Revista Recursos Hídricos, v. 32, p. 45-52, 2011.

Santos, S. T.; Santos, S. M.; Montenegro, S. M. G. L.; Coutinho, A. P.; Moura, G. S. S.; Antonino, A. C. D. Telhado verde: desempenho do sistema construtivo na redução do escoamento superficial. Ambiente Construído, v. 13, n. 1, p.161-174, 2013. https://doi.org/ 10.1590/S1678-86212013000100011

Silva, P. C. G. S.; Moura, M. S. B.; Kiill, L. H. P.; Brito, L. T. L.; Pereira, L. A.; Sá, I. B.; Correia, R. C.; Teixeira, A. H. C.; Cunha, T. J. F.; Guimarães Filho, C. Caracterização do semiárido brasileiro: fatores naturais e humanos. In: Sá, I. B.; Silva, P. C. G. S. Semiárido brasileiro: pesquisa, desenvolvimento e inovação. Petrolina: Embrapa Semiárido, 2010.

SIURB - Secretaria de Infraestrutura Urbana e Obras. Dados dos serviços de manutenção realizados na rede de esgotamento sanitário de Caruaru. Caruaru: Prefeitura Municipal de Caruaru, 2021.

Tsutiya, M. T.; Bueno, R. C. R. Contribuição de águas pluviais em sistemas de esgotosanitário no Brasil. Anais do Seminários Redes de Esgoto Sanitários e Galerias de Águas Pluviais: Interferências e Interconexões, São Paulo, 2004.

Tsutiya, M. T.; Além Sobrinho, P. Coleta e transporte de esgoto sanitário. 3. ed. Rio de Janeiro: ABES, 2011. 
Tsutiya, M. T.; Bueno, R. C. R.; Cintra, E. M.; Reami, L. Contribuição de águas pluviais em sistemas de esgotos sanitários: estudo de caso da Cidade de Franca, Estado de São Paulo. Anais do $22^{\circ}$ Congresso Brasileiro de Engenharia Sanitária e Ambiental, Santa Catarina, 2003.

Vaz, A. J. A importância da rede coletora de esgoto na promoção da qualidade sócioambiental. 2015. Disponível em: <http://observatoriogeograficoamericalatina.org.mx/ egal12/Procesosambientales/Impactoambiental/71.pdf>. Acesso em: 20 jul. 2021.

Vieira, B. F.; Siciliano, B. G.; Cruciani Neto, N.; Goulart, R. M. Impacto da intrusão de águas pluviais na qualidade do esgoto tratado. São Caetano do Sul: Escola de Engenharia Mauá, 2015. (Trabalho de conclusão de curso).

Volschan, I.; Tsutiya, M. T.; Martins, R. H. O.; Yazaki, L. F. O. Sistema unitário x sistema separador absoluto qual o mais atraente para as condições brasileiras? Revista DAE, n. 180 , p. 40-43, 2009.

Von Sperling, M. Introdução à qualidade das águas e ao tratamento de esgotos. Belo Horizonte: Universidade Federal de Minas Gerais, 2005.

Watanabe, J.; Yamamoto, J. K.; Rocha, M. M.; Fonseca, P. P. Estudo da influência da correlação inicial entre variáveis nos resultados de co-estimativas. Geociências, v. 28, n. 4, p. 467-484, 2009.

Informação da Licença: Este é um artigo Open Access distribuído sob os termos da Licença Creative Commons Attribution, que permite uso irrestrito, distribuição e reprodução em qualquer meio, desde que a obra original seja devidamente citada. 